\title{
MIMO-FM: some new experimental results proving the concept feasibility
}

\author{
Dominique Poullin, Olivier Rabaste, Michel Menelle, Bruno Urbani and Maxime Goujon \\ DEMR, ONERA, Université Paris-Saclay \\ F-91123 Palaiseau - France
}

\begin{abstract}
In this paper, we consider the MIMO-FM concept firstly described in [1]. This particular setting enables to retrieve the elevation of targets in FM passive radar by exploiting the presence of multiple transmitting antennas located at different heights on the same pylon and transmitting signals at different frequencies, without the need for a vertical reception array. In [1], due to a bad sensitivity of the receiver used for the experiment, the MIMOFM concept could be only verified for very close targets. We review here the main features of this concept and in particular of the specific processing it requires. We then present new results obtained with an improved receiver that enables to detect targets at more than $100 \mathrm{~km}$ in bistatic range, thus proving the feasibility of the MIMO-FM concept for a various set of targets of configurations.
\end{abstract}

\section{INTRODUCTION}

An FM passive radar exploits FM (Frequency Modulation) transmitters present in the surrounding neighbourhood in order to detect possible targets of interest in the vicinity of the receiver [2], [3], [4]. One main advantage of such a system is that it does not radiate any power and is thus naturally lowcost. Besides, since the FM transmitters are generally omnidirectional and quite powerful, this passive system presents a wide surveillance coverage area.

In classic surveillance applications, it is of interest to retrieve different target parameters such as the range and the Doppler shift, as well as the azimut and elevation angle. In order to retrieve these two last parameters, a classic system will consider a reception array spread in the horizontal and vertical dimensions. In passive systems exploiting Digital Video Broadcasting (DVB) signals [5], a vertical array could be considered in order to retrieve the target elevation, thanks to transmitted frequencies ranging between 500 and $800 \mathrm{MHz}$ [6]. However, in the case of FM signals, the classic carrier frequencies range between 88 and $108 \mathrm{MHz}$, thus corresponding to an approximate wavelength of $3 \mathrm{~m}$. With such a wavelength, retrieving the elevation parameter with a good accuracy would require a very high reception array. This is clearly not appropriate for practical purposes.

The authors have proposed recently a different solution [1], based on the coherent colocated MIMO radar framework [7], [8], [9]. With a coherent MIMO radar, each transmitting antenna transmits a different code so that the resulting signal transmitted by the whole array is not the same in two different directions: the space has been coded. Thanks to this particular space coding feature, a dedicated processing at the reception enables to retrieve the angular information, even if no angular estimation capability is provided by the reception array. In the solution [1], we thus proposed to retrieve the elevation estimation by exploiting the particular geometry of some FM transmitters and their specific frequency coding: for instance, FM transmitters present at the top of the Eiffel Tower in Paris are located at different heights and transmit separate frequency bands. This new MIMO-FM solution was tested on a few real data recordings, showing that the elevation angle could be indeed estimated to some extent with this procedure. However, due to the poor quality of the receiver used for the data acquisition, only a few close targets (at most located at $15 \mathrm{~km}$ in bistatic range) could be detected and processed by the MIMO-FM specific processing. Since these targets were all following the same trajectories and were all at low elevation angles, it was not possible to check the feasibility of the MIMO-FM processing in general configurations. In particular, the MIMO-FM ambiguity function is subject to very strong ambiguities that could not be observed on this preliminary set of measurements.

Based on these observations, we decided to change our MIMO-FM receiver in order to obtain more detections on various target trajectories with larger ranges (more than $100 \mathrm{~km}$ in bistatic range) and various elevation angles. This new receiver indeed provided us many detections that enabled to validate the MIMO-FM principle for a large variety of trajectories and elevation angles. The new recorded data also highlighted the strong ambiguity phenomenom that this MIMO-FM processing may face according to the specific configuration (in terms of transmitting antenna heights, and transmitted carrier frequencies for each antenna). We provide in this article several examples of such ambiguities. Some forthcoming work will focus on the use of some possible tricks to mitigate, when possible, these ambiguities.

This paper is organized as follows: in section II, we recall the general expression for the received signal, the corresponding MIMO-FM ambiguity function and the proposed MIMO-FM processing that enables target elevation estimation. In section III, we present our experimental setting and the transmitter configuration. In section IV we present different experimental results.

\section{MIMO-FM AMBIGUITY FUNCTION AND SPECIFIC PROCESSING}

In this section, we will recall the general form of the MIMO-FM ambiguity function and the resulting specific processing as presented in [1]. Notations will be the same as in [1]. 


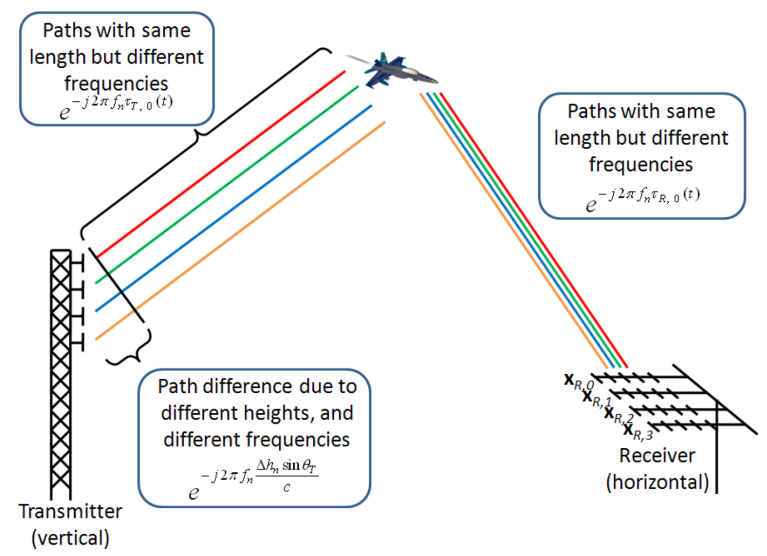

Fig. 1. Configuration for the MIMO-FM passive radar.

Let us consider the geometrical setting presented in Figure 1 composed of a vertical transmitting array and a horizontal receiving array. Let us denote by $\left(h_{n}\right)_{n \in\left\{0, \ldots, N_{T}-1\right\}}$ the heights of the $N_{T}$ FM transmitting antennas, all located on the same pylon. We will denote by $\Delta h_{n}=h_{n}-h_{0}$ the relative height between the reference transmitter located in $h_{0}$ and the $n^{\text {th }}$ transmitter, and by $f_{n}$ the carrier frequency used to carry the baseband FM signal $s_{n}(t)$ transmitted by antenna $n$. As for the receiving array, it is assumed to be composed of $N_{R}$ antennas at positions $\left(\mathbf{x}_{R, m}\right)_{m \in\left\{0, \ldots, N_{R}-1\right\}}$.

With such notations, the received signal for a target with bistatic delay $\tau_{0}=\tau_{E, 0}+\tau_{R, 0}$ where $\tau_{E, 0}$ represents the delay between the $n=0$ reference antenna of the transmission array and the target and $\tau_{r, 0}$ represents the delay between the target and the $m=0$ reference antenna of the reception array, bistatic velocity $v_{0}$, direction vector $\mathbf{u}_{R}$ between the target and the reception array, and elevation angle $\theta_{T}$ with respect to the transmission array can be written, under classic Doppler assumptions, as [1]:

$$
\begin{aligned}
& s_{R, m}(t)=\sum_{n=0}^{N_{T}-1} s_{n}\left(t-\tau_{0}\right) e^{j 2 \pi f_{n}\left(t-\tau_{0}\right)} \\
& \times e^{-j 2 \pi f_{n} \frac{v_{0}}{c} t} e^{-j 2 \pi f_{n} \frac{\Delta h_{n} \sin \theta_{T}}{c}} e^{j 2 \pi f_{n} \frac{\mathbf{x}_{R, m}^{T} \mathbf{u}_{R}}{c}} .
\end{aligned}
$$

In this equation appear different phase terms that correspond to different phenomena:

- phase term $e^{j 2 \pi f_{n}\left(t-\tau_{0}\right)}$ accounts for the phase shift due to the reference target delay that differs from transmitter to transmitter because of the different frequencies transmitted.

- $\quad$ phase term $e^{-j 2 \pi f_{n} \frac{v_{0}}{c} t}$ accounts for the phase shift due to the bistatic velocity of the target. Again it differs from transmitter to transmitter because of the different frequencies exploited, so that the parameter to consider must be the target velocity and not simply its Doppler shift.

- $\quad$ phase term $e^{-j 2 \pi f_{n} \frac{\Delta h_{n} \sin \theta_{T}}{c}}$ corresponds to the phase shift on the transmitting array due to the target elevation.

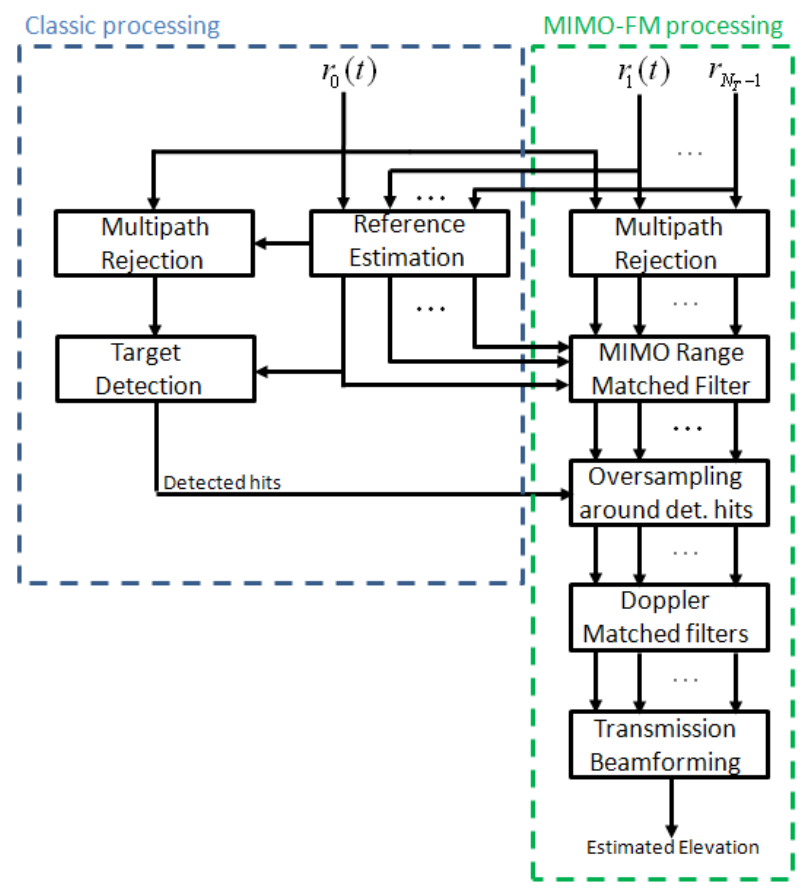

Fig. 2. Proposed MIMO-FM processing. For simplicity, reception beamforming does not appear here.

- phase term $e^{j 2 \pi f_{n} \frac{\mathbf{x}_{R, m}^{T} \mathbf{u}_{R}}{c}}$ finally accounts for the phase shift measured on the reception array due to the target azimut angle.

Detecting the target and estimating its bistatic delay $\tau$, bistatic velocity $v$, elevation angle $\theta$ and azimut angle $\mathbf{u}$ can be performed using the classic matched filter approach, that consists in correlating the received signal with theoretical replicas of the target signal computed for all possible combination of these parameters. The output of such a matched filter corresponds to the MIMO-FM ambiguity function which is a function of $(\tau, v, \theta, \mathbf{u})$ provided in Equation (2) [1].

$$
\begin{aligned}
& A(\tau, v, \theta, \mathbf{u})=\sum_{n=0}^{N_{T}-1} \sum_{m=0}^{N_{R}-1} e^{-j 2 \pi f_{n}} \frac{\Delta h_{n}\left(\sin \theta_{T}-\sin \theta\right)}{c} e^{j 2 \pi f_{n} \frac{\mathbf{x}_{R, m}^{T}\left(\mathbf{u}_{R}-\mathbf{u}\right)}{c}} \\
& \times \int s_{n}\left(t-\tau_{T}\right) s_{n}^{*}(t-\tau) e^{-j 2 \pi f_{n}\left(\tau_{T}-\tau\right)} e^{-j 2 \pi f_{n} \frac{v_{T}-v}{c} t} d t
\end{aligned}
$$

This expression is computed under the assumption that the different transmitted signals $s_{n}(t)$ are orthogonal, i.e.

$$
\int s_{n}(t) s_{m}(t) d t=0, \forall n \neq m \text {. }
$$

In the MIMO-FM setting, this assumption is made valid whenever the different carrier frequencies are separated by more than the bandwidth of one FM channel, i.e. they verify $\left|f_{n}-f_{m}\right|>B_{F M}$ where $B_{F M}$ is the bandwidth of an FM channel. This is generally verified by the different radio channels, and it thus suffices to consider for each $s_{n}(t)$ a different radio channel.

Clearly the MIMO-FM matched filter consists in coherently compensating each phase term previously described, 
as for a classic radar processing. However due to the presence of different frequencies, the order in which the different processing steps are performed cannot be inverted, i.e. the reception processing corresponding to phase term $e^{j 2 \pi f_{n} \frac{\mathbf{x}_{R, m}^{T}\left(\mathbf{u}_{R}-\mathbf{u}\right)}{c}}$ and the Doppler processing corresponding to phase term $e^{-j 2 \pi f_{n} \frac{v_{T}-v}{c} t}$, that both involve phase terms depending on the transmitted frequencies, must be performed before the transmission processing corresponding to phase term $e^{-j 2 \pi f_{n} \frac{\Delta h_{n}\left(\sin \theta_{T}-\sin \theta\right)}{c}}$. Besides the classic Doppler processing performed via a simple FFT (Fast Fourier Transform) over a set of subpulses obtained by splitting the received signal into several subpulses is not ideal here, since the Doppler frequency shift involved depends on the different carrier frequencies. Such a use of the FFT would require afterwards to interpolate the output in order to retrieve the same target velocity. It seems more simple to directly compensate, for each considered frequency, the corresponding phase vector. Finally, note that very strong ambiguities are created by the presence of these many phase terms, and in particular the product $e^{-j 2 \pi f_{n} \tau_{0}} e^{-j 2 \pi f_{n} \frac{\Delta h_{n} \sin \theta_{T}}{c}}$ that involves the different frequencies.

An interesting by-product of the MIMO-FM processing is the increase in the overall signal bandwidth considered. A classic single FM channel presents a very small bandwidth (at most $75 \mathrm{kHz}$ ) and thus a poor range resolution. On the contrary, in the MIMO-FM, since several different frequencies are considered, the overall bandwidth exploited in the processing ranges between $\min _{n}\left(f_{n}\right)$ and $\max _{n}\left(f_{n}\right)$, and can thus span up to 1 or $2 \mathrm{MHz}$, thus potentially providing a much interesting range resolution. However this implies that received signals must be dramatically oversampled to cope with this full bandwidth and enable the elevation estimation, thus increasing the computational cost of the method. This MIMO-FM cannot thus realistically be performed on the full signal duration. Preferably, a classic passive processing should be applied to one predefined frequency to perform target detection, and then only should the MIMO-FM processing be performed on the detected hits to perform the elevation estimation.

The MIMO-FM processing can then be performed as follows:

- Classic processing:

- signal reference estimation based on reception beamforming;

- multipath rejection, for instance by least square method [10];

- target detection, producing estimated hits.

- MIMO-FM specific elevation estimation processing:

- signal reference estimation for considered transmitted frequencies;

- multipath rejection for each considered frequencies;

- range matched filter for each frequency channel with the corresponding reference signal;

o oversampling of the output of the range matched filter around each detected hits provided by the classic processing;

- specific Doppler matched filtering based on target velocity;

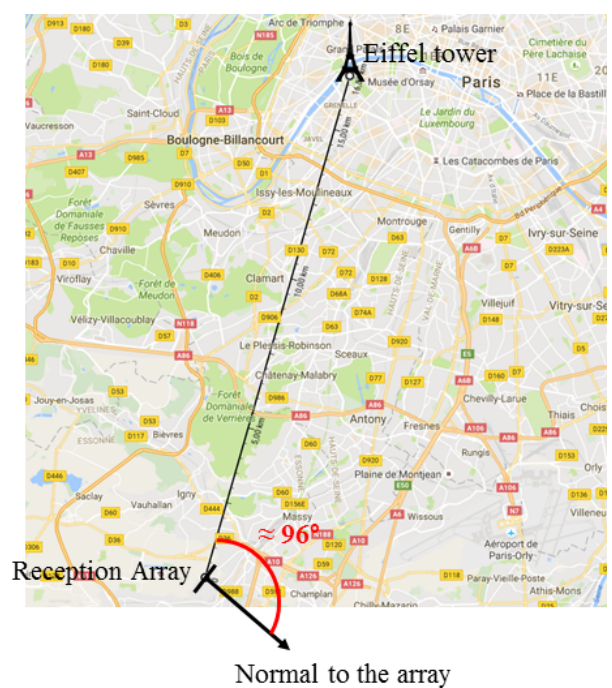

Fig. 3. Configuration of the experimental setting. The reception device is located in Palaiseau in the South of Paris. The Eiffel tower used as transmitter of opportunity is located in a direction of approximately $96^{\circ}$ with respect to the normal of the reception array and at a distance of approximately $16.8 \mathrm{~km}$ from the reception array.

- specific MIMO-FM transmission beamforming.

Finally the overall processing diagram presenting this MIMOFM processing is provided in Figure 2 [1].

\section{EXPERIMENTAL SETTING}

First MIMO-FM data presented in [1] had been recorded during Spring 2017 with a deficient receiver with very limited sensitivity only enabling to detect very close targets (airplanes landing at Orly airport at only a few kilometers in bistatic range). Data that will be presented thereafter have been recorded at the beginning of 2019 with an improved receiver device enabling to detect targets at much larger range (more than $100 \mathrm{~km}$ in bistatic range), and a somehow different configuration. As before, the considered FM transmitter is the Eiffel Tower, where several FM antennas are deployed at the top of the Tower, at 4 different heights. The reception array is located at the top of one ONERA building at Palaiseau near Paris. The configuration is presented in Figure 3 . It is composed of 6 antennas and can record up to six different frequency channels. For the measurements, the 6 frequency bands recorded were $102.7,103.1,102.3,101.9,103.9$ and 103.5 $\mathrm{MHz}$, and the corresponding transmitting antennas on the Eiffel Tower are respectively located at heights 291, 294, 299, 304, 294 and $304 \mathrm{~m}$ above ground. In this new experimental setting, the normal of the reception array was roughly directed towards the East while North (and thus the Eiffel tower) was roughly in the axis of the array. Cancellation of the direct path, approximately located at an angle of $96^{\circ}$ with respect to the antenna array, thus induced a bad azimut estimation for targets located approximately at $96^{\circ} \pm 30^{\circ}$ as we will observe in the following figures. Finally a partial ground truth was obtained thanks to an ADSB receiver recording information from surrounding airplanes. 

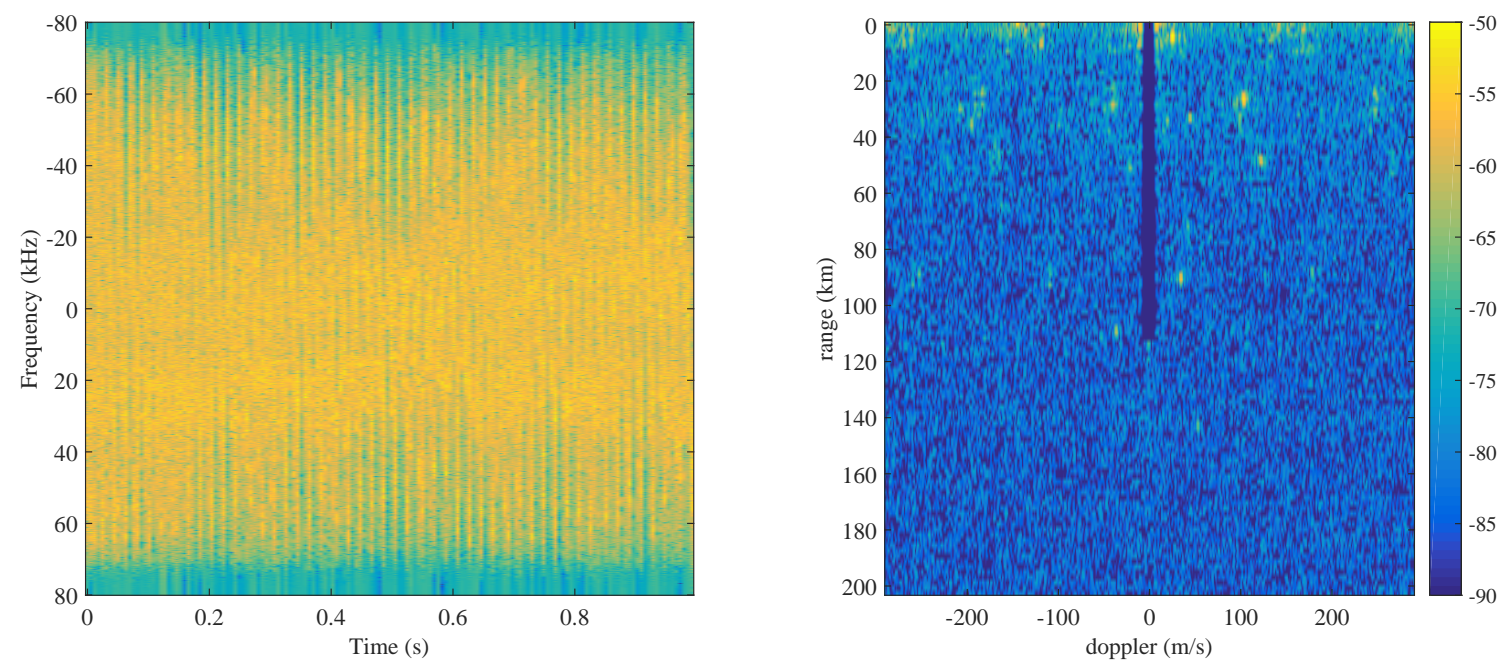

Fig. 4. Left: Spectrogram of the signal used for detection during the integration time (equal to 1s). Frequencies are indicated in baseband with respect to the central frequency of the channel which is here equal to $103.9 \mathrm{MHz}$. Right: Range-Doppler map after the rejection step for an integration time of 1s. Many targets are clearly visible at least up to $100 \mathrm{~km}$ range.

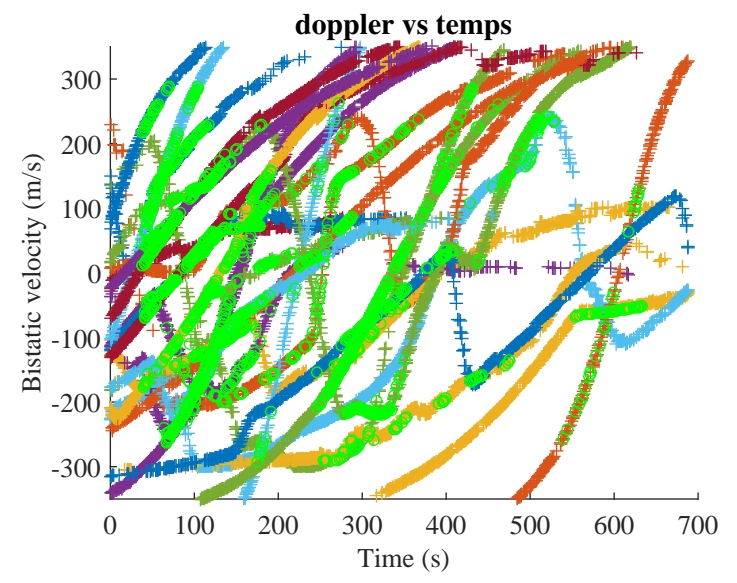

Fig. 5. Doppler evolution of detected tracks (green circle $\circ$ ) matching the recorded ADSB tracks (plus + ) for a 10 min recording.

\section{EXPERIMENTAL RESULTS}

As mentionned in section II, the first step in the MIMOFM processing consists in the detection step applied to only one single frequency. Here the frequency used for detection was $f_{c}=103.9 \mathrm{MHz}$ because it presented the best data quality in terms of bandwidth occupancy during the $10 \mathrm{~min}$ record duration. This detection procedure consists in the classic passive radar processing, i.e. estimation of the reference signal, rejection of fixed echoes at zero Doppler (direct path and multiple paths), and application of a detection threshold on the resulting range-Doppler map. Such a range-Doppler map is presented in Figure 4.

The output of the detection step is provided in Figure 5 and 6 where we present the doppler estimates and the range estimates with respect to time of the detected hits that were mapped to existing ADSB tracks.

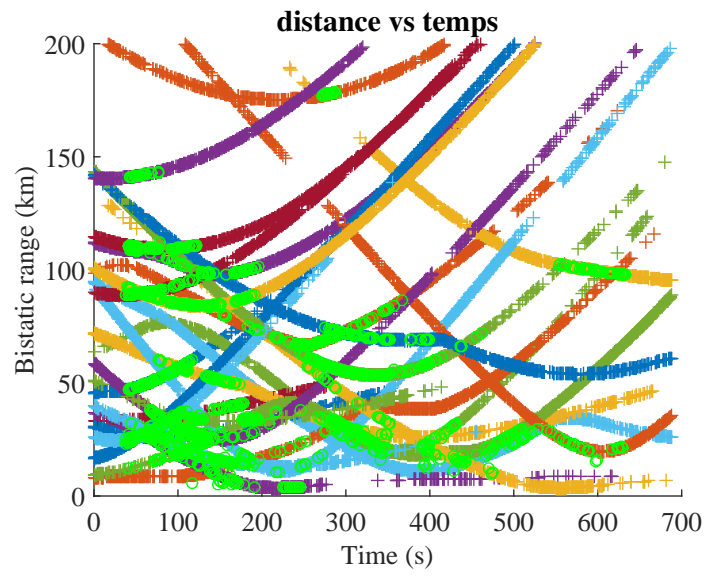

Fig. 6. Distance evolution of detected tracks (green circle $\circ$ ) matching the recorded ADSB tracks (plus + ) for a 10 min recording.

Once the detected hits have been formed, it is then possible to apply the MIMO-FM processing on each hit, exploiting all the 6 frequencies recorded by the reception device. Results are presented here for two different tracks in Figure 9 and 10. For each track considered, we present here the estimation result for the four parameters of the MIMO-FM ambiguity function, i.e. the range, the Doppler shift, the elevation angle and the azimut angle. In Figure 9, all four parameters have been correctly estimated when the target has been detected, in the sense that the estimated values match the ADSB track. On the contrary, in Figure 10, although the range, Doppler shift and azimut parameters have been correctly estimated, the elevation parameters appear to present strong biases for some estimates along time, that are gathered at two distinct values. These biases are due to the presence of the strong ambiguity lobes in the MIMO-FM ambiguity function. Note also that we can observe in Figure 9 that the elevation estimates correctly follow the evolution (that increases) of the target along time. 


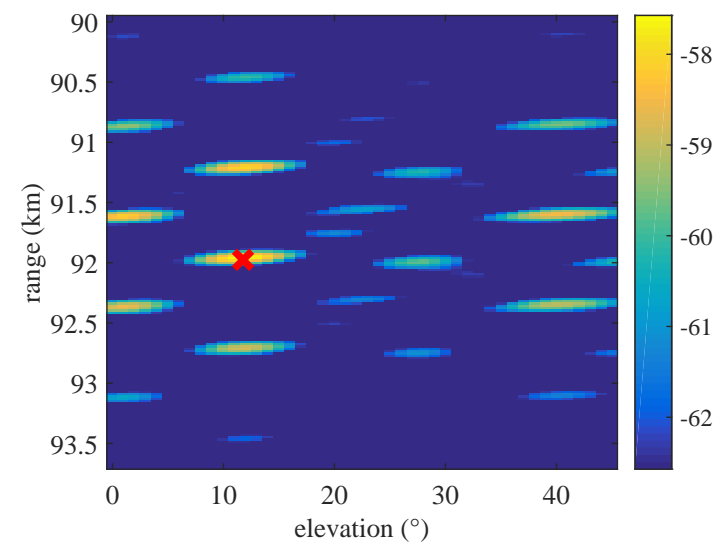

Fig. 7. MIMO-FM ambiguity function of hit n.5 presented in Figure 10. The red cross indicates the position of the maximum level of the ambiguity function. The estimation has been performed correctly. Note that the measured level for the good position at elevation around $11^{\circ}$ is equal to $-57.57 \mathrm{~dB}$ while the level for the strongest ambiguity at elevation around $40^{\circ}$ is equal to $-58.46 \mathrm{~dB}$, which represents a difference of $0.89 \mathrm{~dB}$.

A careful look at the MIMO-FM ambiguity function indeed shows that the estimator can easily pass from the true value to one of the ambiguity ones. We present in Figure 7 the range/elevation cut of the ambiguity function measured for a hit where the estimation has been done correctly, and in Figure 8 the range/elevation cut of the ambiguity function measured for a hit where the estimation has been biased by an ambiguity. In Figure 7, the maximum of the ambiguity function corresponds to the good parameter value, and the level difference between this maximum value and the level of the strongest ambiguous sidelobe providing a biased elevation estimate is equal to $0.89 \mathrm{~dB}$. In Figure 8, the maximum of the ambiguity function does not correspond to the good parameter value, but is located at a strong ambiguous sidelobe. The level difference between this ambiguous sidelobe and the level of the good parameter lobe is very small and equal here to only $0.15 \mathrm{~dB}$. Clearly both ambiguity function look pretty similar, but since the level difference in both cases is very small, a small noise difference can easily make one of the ambiguous lobe become more energetic than the good mainlobe. Note also that these ambiguity functions easily explain the two type of biases observed in Figure 10 around $40^{\circ}$ and around $25^{\circ}$. We indeed observe strong sidelobes in the MIMO-FM ambiguity function that arise at both elevations, although the sidelobes at $25^{\circ}$ are weaker (a few $\mathrm{dB}$ at most), at least in these examples. Furthermore, these ambiguity functions may be affected by some frequency components that may be temporally unefficient due to locally low bandwidth occupancy. Finally we can notice that the targets are detected only on part of their trajectory. This may be explained either by a maximum detection range with respect to their respective SER (case of Figure 10 for instance)

\section{CONCLUSION}

In this article, we have reviewed the MIMO-FM concept first presented in [1]. In this previous article, the proof of feasibility could not be achieved in the general case because of a very bad sensitivity of the receiver used for the experimenta-

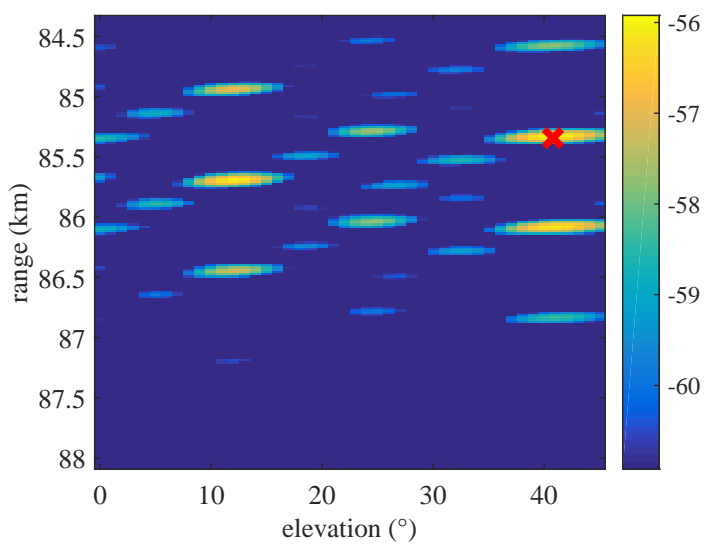

Fig. 8. MIMO-FM ambiguity function of hit n.26 presented in Figure 10 The red cross indicates the position of the maximum level of the ambiguity function. The estimation has not been performed correctly: the maximum of the ambiguity function is located on an ambiguity. The measured level for the good position at elevation around $11^{\circ}$ is equal here to $-56.07 \mathrm{~dB}$ while the level for the strongest ambiguity at elevation around $40^{\circ}$ is equal to -55.92 $\mathrm{dB}$, which represents a difference of only $0.15 \mathrm{~dB}$.

tion, that enabled to detect only very close targets (at most 15 $\mathrm{km}$ in bistatic range), almost all presenting the same elevation. Since then, we have considered a new receiver which provides a much better sensitivity and enables to detect targets at more than $100 \mathrm{~km}$ in bistatic range. Experimentations performed with this new receiver allowed to validate the feasibility of the MIMO-FM concept for a wider class of targets, including different elevations and in particular to check the ability to follow the dynamic in elevation of a target.

\section{REFERENCES}

[1] D. Poullin, O. Rabaste, M. Lesturgie, M. Menelle, and S. Azarian. MIMO-FM: A solution to estimate target elevation with passive FM radar. In 2018 International Conference on Radar, 2018.

[2] B.D. Nordwall. "Silent sentry" A new type of Radar. Aviat. Week Space Technol., pages 70-73, 1998.

[3] N.J. Willis and H.D. Griffiths. Advances in Bistatic Radar. Scitech Publishing, Raleigh, NC, 2007.

[4] P.E. Howland, D. Maksimiuk, and G. Reitsma. FM radio based bistatic radar. IEE Proc. Radar Sonar Navig., 152(3):107-115, 2005.

[5] D. Poullin. Passive Detection using digital broadcasters (DAB, DVB) with COFDM modulation. IEE Proc. Radar Sonar Navig., 152(3):143152, 2005.

[6] D. Poullin, M. Flécheux, and M. Klein. Elevation angle estimation for low-altitude targets using DVB (SFN broadcasters). IEEE Aerospace and Electronic Systems Magazine, 27(11):27-35, 2012.

[7] J. Li and P. Stoica. MIMO Radar with Colocated Antennas. IEEE Signal Processing Magazine, pages 106-114, 2007.

[8] C.Y. Chen and P.P. Vaidyanathan. MIMO Radar Ambiguity Properties and Optimization Using Frequency-Hopping Waveforms. IEEE Trans. on Signal Processing, 56(12):5926-59368, 2008.

[9] Y.I. Abramovich, G.J. Frazer, and B.A. Johnson. Transmit and receive antenna array geometries for mode selective HF OTH MIMO radar. In 18th European signal Processing Conference, pages 1244-1248, 2010.

[10] F. Colone, D.W. O'Hagan, P. Lombardo, and C.J. Baker. A Multistage Processing Algorithm for Disturbance Removal and Target Detection in Passive Bistatic Radar. IEEE Transactions on aerospace and electronic systems, 45(2):698-722, 2009. 

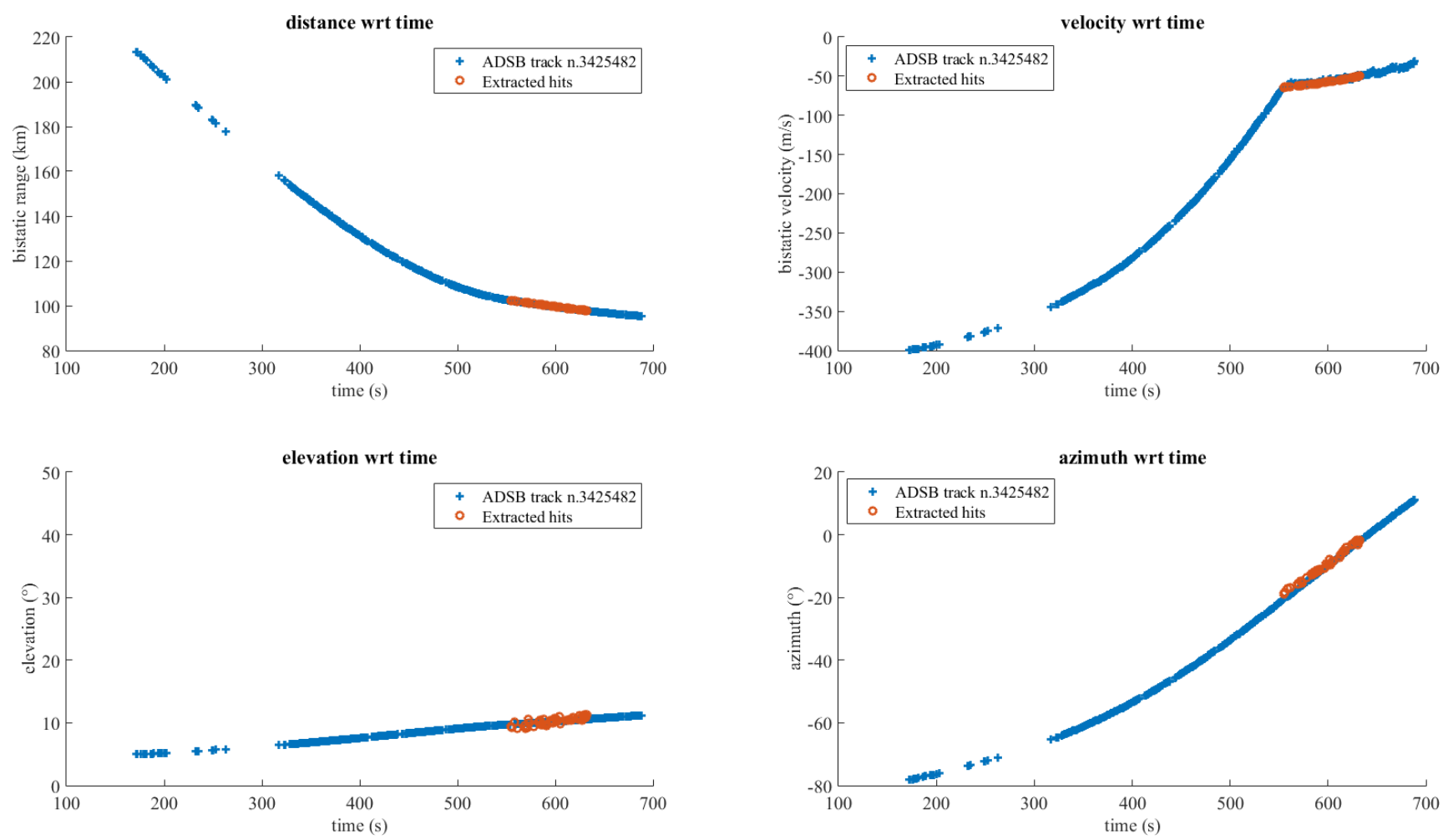

Fig. 9. Estimation results for one ADSB track. The estimation has performed well for the four parameters considered (range, Doppler, elevation angle, azimut angle).
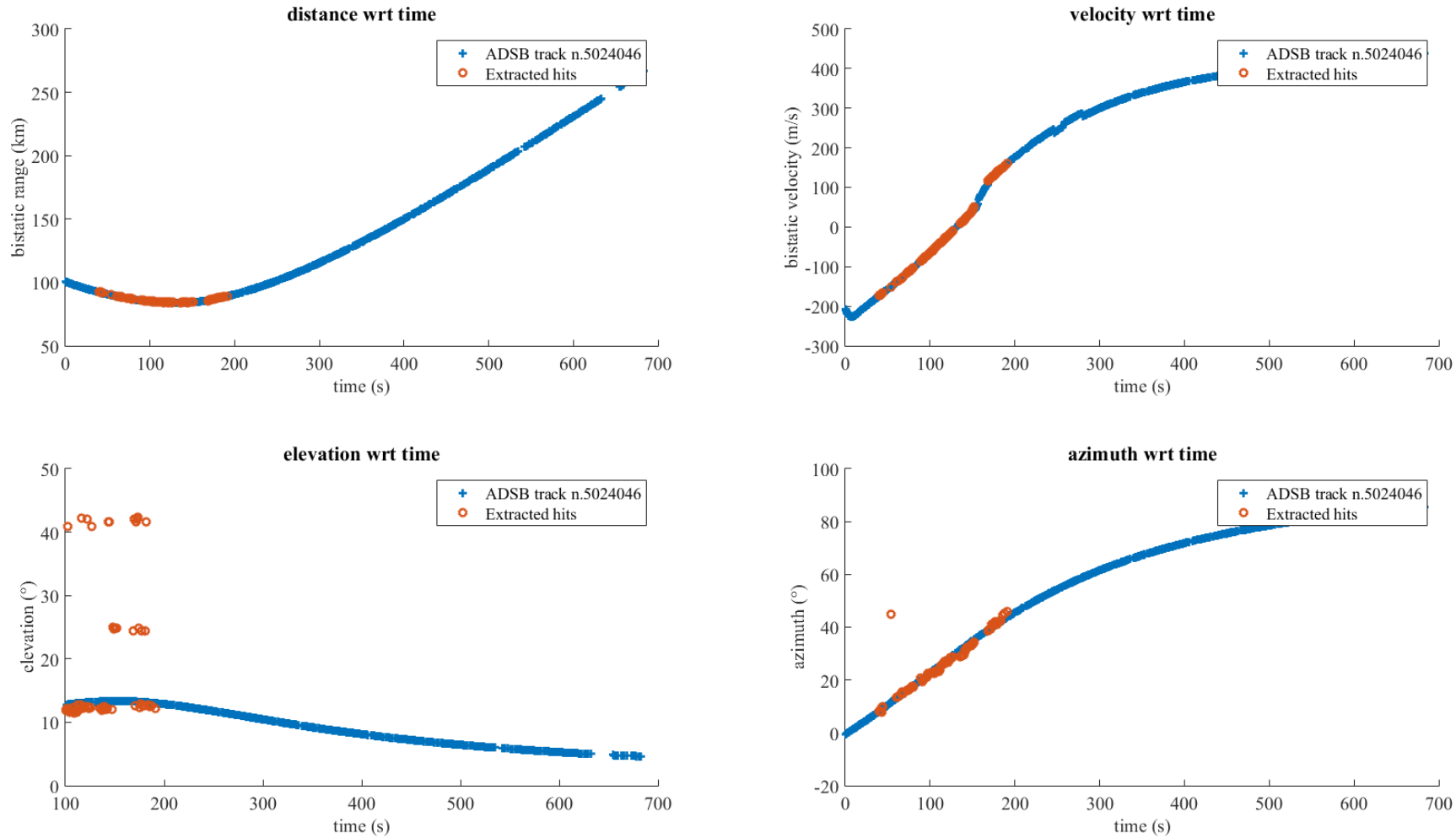

Fig. 10. Estimation results for another ADSB track. The estimation has performed well for three of the four parameters considered (range, Doppler, azimut angle). For the elevation angle, the estimator has sometimes been trapped to one ambiguity of the MIMO-FM ambiguity function, thus leading to a biased estimator. 